\title{
The Management of Congenital Malpositions of Eyelids, Eyes and Orbits
}

\author{
S. MORAX AND T. HURBLI
}

Paris

\begin{abstract}
Summary
Congenital malformations of the eye and its adnexa which are multiple and varied can affect the whole eyeball or any part of it, as well as the orbit, eyelids, lacrimal ducts, extra-ocular muscles and conjunctiva.

A classification of these malformations is presented together with the general principles of treatment, age of operating and surgical tactics. The authors give some examples of the anatomo-clinical forms, eyelid malpositions such as entropion, ectropion, ptosis, levator eyelid retraction, medial canthus malposition, congenital eyelid colobomas, and congenital orbital abnormalities (Craniofacial stenosis, orbital plagiocephalies, hypertelorism, anophthalmos, microphthalmos and cryptophthalmos).
\end{abstract}

Congenital malformations of the eye and its adnexa are multiple and varied. They can affect the whole eyeball or any part of it, as well as the orbit, eyelids, lacrimal ducts extraocular muscles and conjunctiva.

From the anatomical point of view, the following can be considered.

Position abnormalities (malpositions) of one or more elements and formation abnormalities (malformations) of the same organs.

Some of these abnormalities are limited to one organ and can be subjected to a relatively simple and well recognised surgical treatment. But often they are more widespread, involving eye and adnexa, oculo-facial region or eye, face and other organs, in some well known malformative syndrome.

The clinical work-up must always include a description of the presenting signs and symptoms and an investigation of other signs through a careful ophthalmic and systemic examination.

As a result of the clinical findings, some investigations will then be carried out: radiologic work-up ranging from simple $\mathrm{X}$-Ray to more sophisticated techniques such as echography, CT-scan and NMR, enzymatic work-up or genetic studies (Table I).

Surgical treatment when feasible will encounter numerous problems; age will play a role, choice of a surgical protocol directly related to the existing complaints, and cooperation between several surgical teams (ophthalmologic, plastic, cranio-maxillo-facial and neurosurgical), the ideal being to treat

Table I The management of congenital malpositions of eyelids, eyes and orbits

Ocular Findings:

Eyeball, eyelids, lacrimal passages, orbit, socket, motility.

Family History

Systemic Manifestations:

Face

General

Radiologic investigations:

Echography, X-Rays, CT-Scan, NMR

Laboratory investigations.

Genetic transmission and counselling. 
the maximum amount of abnormalities in a single setting, hence decreasing the number of surgical interventions that will be needed.

The mechanism of the congenital ophthalmologic disorder may be direct, in this case the symptom can either be isolated or associated with a more complex malformation creating syndromes or diseases. On the other hand, because of the intricate relationships between the eye and its adnexa, some disorders may, indirectly, be a consequence of a pre-existing malformation. For instance, congenital microphthalmos may result in microorbitism that will need a clinical and radiological work-up. Similarly, a severe craniostenosis will lead to malposition of the eyeball (exophthalmos), this secondary exophthalmos may induce corneal exposure and keratitis. As no single symptom of the ocular area is pathognomonic alone, it is very important to describe precisely each symptom and link it to its true cause or mechanism.

\section{Classification}

A number of classifications have been proposed according to the type of lesion or tissue involvement (soft tissue only, combined soft tissue and underlying bone or indirect involvement of soft tissue as a result of bony malformation).

We prefer a classification which relates to the involved organ (Table II).

\section{A-Eyeball Abnormalities}

These can be of size or of shape. Microphthalmos or anophthalmos are very precise examples. True anophthalmos is rarely encountered and CT-scan often reveals that in fact it is simply a severe microphthalmos with sometimes a colobomatous cyst. These isolated malformations can also be associated in more complicated syndromes, e.g. first branchial arch or facial cleft syndromes. The small size of an unaesthetic and non functional eyeball will result in lack of development of the bony orbit, producing a relative microorbitism and lid size reduction ranging from simple microblephary to subtotal ablephary.

\section{B-Position Abnormalities}

Ocular dystopias, are displacement of the eyeball in a vertical, transverse or sagittal plane

\section{Table II Ocular findings}

EYE Vision, visual fields, intraocular pressure, anterior segment and sclera, retina and choroid optic nerve, size and malformations (anophthalmos, microphthalmos, cryptophthalmos), malpositions: ocular dystopia, motility: muscular agenesis, muscular extorsion superior oblique fontalisation.

SOCKET Dermoids, dermolipomas, lipomas, dermatocoeles pterygoides ( $=$ choristomas) Investigation for 1st brachial arch syndrome and facial cleft.

EYELIDS (a) Eyelid margin malpositions:

- Congenital entropion/epiblepharon

- Congenital ectropion.

(b) Levator dysfunction (neurogenic, myogenic):

- Ptosis

- Eyelid retraction.

(c) Medial canthus malpositions (epicanthus, telecanthus, telorbitism.

(d) Lateral canthus malpositions: antimongoloid fissures.

(e) Colobomas and ablephary.

LACRIMAL DUCTS ABNORMALITIES

Colobomas, stenosis ...

CONGENITAL ORBITAL ABNORMALITIES

Micro/Macro—orbitism—craniofacial stenosis, plagiocephal-telorbitism.

often as a result of congenital bony or orbital contents abnormalities as encountered in craniostenosis or Von Recklinghausen's disease. The eye may stay normally formed and functional, the corneal reflexes well centred and the motility normal. Strabismus is not found in all cases. Treatment must include repositioning of the bony orbit.

\section{C-Oculomotor Abnormalities in Orbital Malformations}

These may be isolated or associated with other abnormalities. Direct lesions of the muscles occur: agenesis (as the agenesis of the superior rectus in Apert syndrome), or malposition of the muscle insertion or direction due to orbital malposition (often extorsion). The most frequent syndrome is macular pseudo-ectropia with extorsion of all four recti that act like oblique muscles, resulting in a V-syndrome, a double upshoot and weakness of both superior obliques. This is most 
frequently encountered in hypertelorism and Treacher-Collins syndrome with antimongoloïd lids.

Hypertropia by hyperaction of the inferior oblique and weakness of the superior oblique can be explained by excessive sagittalisation of the inferior oblique or frontalisation of the superior oblique, as in cases of plagiocephaly where the frontal bone is less developed and retruded as compared to the maxillary.

Exotropia is frequent in hypertelorism, being sometimes the result of the divergence of orbital axes and can be reduced simply by repositioning of the orbit, although muscle surgery may sometimes be needed to correct any residual strabismus.

\section{D-Abnormalities of the Conjunctival Fornices}

The most frequently encountered lesions are those belonging to the groups: dermoïds, dermo-lipomas, dermatocoeles and pterygoides. All can be classified as choristomas (presence of a normal tissue in an unusual location) (Fig. 1a and b).

These non-evolving lesions must incite the search for a first brachial arch syndrome or facial cleft. They can also be associated with palpebral abnormalities (colobomas).

\section{E-Palpebral Malpositions and Congenital Malformations of the lids}

Several categories can be described:

Congenital malpositions of the lid margin:

- Congenital entropion and epiblepharon due to lack of adherence of the inferior lid retractor of the skin.

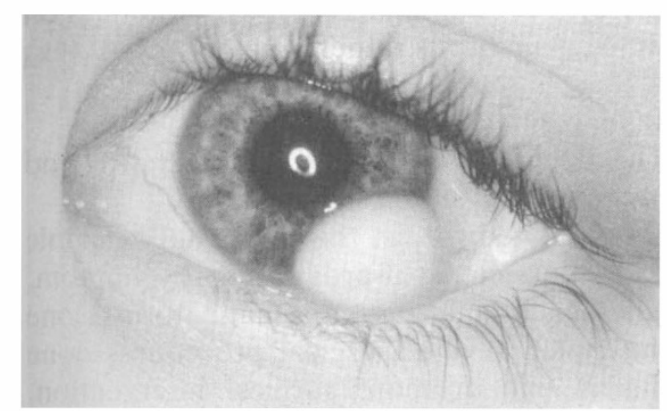

Fig. 1. a-Congenital limbal dermoid.
- Congenital ectropion due to cutaneous lack is either present alone or belongs to a blepharophimosis syndrome.

Levator muscle dysfunction by excess or by deficiency of neurogenous origin or muscular dystrophy.

This gives rise to the congenital ptoses and muscular retractions.

The following elements will be examined: primary position, muscular function, presence of syntines and associated signs.

- Medial canthus malpositions include:

epicanthus, telecanthus and intercanthal space widening by soft tissue lesion. They can either be isolated or integrated in a blepharophimosis or Waardenburg syndrome.

Hypertelorism (Grei syndrome) is an intercanthal widening by lesion of the bony parts, its degree is variable and two aspects are encountered: hypertelorism by simple craniostenosis and hypertelorism by orbito-facial cleft. ${ }^{1,2}$

- Lateral canthus malpositions are less frequent.

The most encountered aspect is the antimongoloid fissure as seen in the Franceschetti-Treacher-Collins syndrome and in telorbitism with extorsion.

- Colobomas and ablephary.

As well as the rest of the face, lids can be subject to clefts. Colobomas are either isolated or associated and belong to the facial cleft group (Fig. 2). One must therefore look

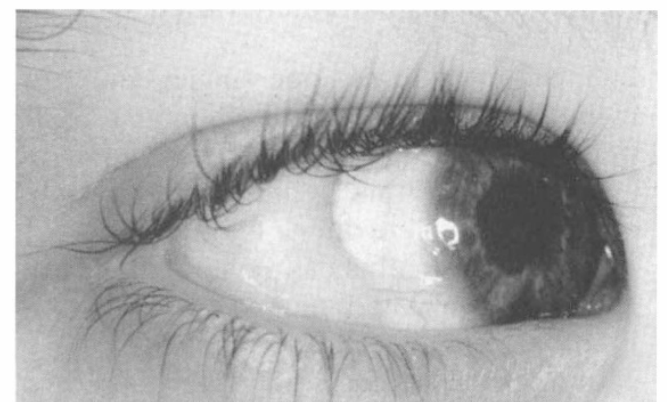

b-Congenital dermolipoma of the lateral canthus (same patient). 


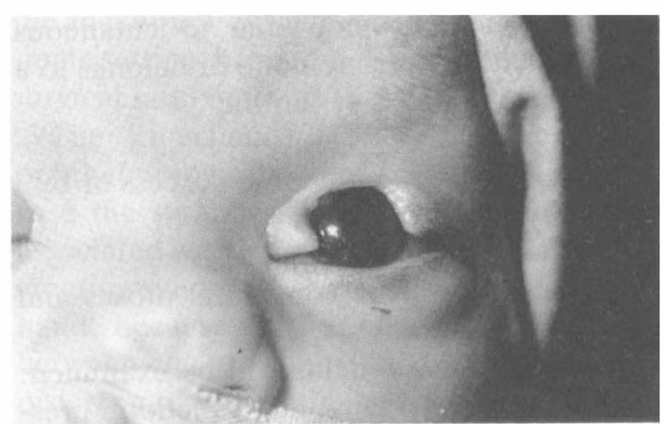

Fig. 2. Congenital superior eyelid coloboma.

for all associated anomalies of a half-axis or a different axis according to Tessier's classification, ${ }^{2}$ on skin, skeleton and lacrimal ducts.

\section{F-Congenital Orbital Abnormalities}

One can describe abnormalities in size: microorbitism related to microphthalmos, macroorbitism as encountered in mesodermal dysplasia with destruction of the greater wing of the sphenoïd bone in von Recklinghausen's disease, and malformative and position abnormalities as dystopias in all three planes: plagiocephaly in the vertical plane, telorbitism in the transverse plane and cranio-facial stenosis in the sagittal plane.

These primary or secondary bony malformations induce sequellae of the visual apparatus: positional sequellae (enophthalmos, exophthalmos); strabismus or visual sequellae: optic nerve compression and corneal exposure.

To summarise, all congenital malformations of the visual apparatus described in each organ can either be found alone or associated and integrated into other diseases; the clinical presentation being extremely varied.

- Isolated forms: only one single symptom calls for treatment, orbital, ocular, palpebral, lacrimal or muscular.

- Associated forms that stay purely ophthalmic, where one or two symptoms of the visual apparatus can be either directly or indirectly related.

- Ophthalmic and facial associated forms.

- More generalised associated forms where ophthalmic, facial and general abnormalities can combine.

\section{General Principles of Treatment}

The severe bony malformations which require cranio-facial surgery have become better classified and more easily treatable since the work of Tessier ${ }^{1.2}$ in the last 20 years. The interest in the treatment of congenital malformations of the visual apparatus lies more in the extremely variable clinical work up and the surgical management of the associated malformations rather than in the purely technical aspect of the surgery.

The plastic surgeon will be faced with several problems:

\section{At what age should one operate?}

Except for emergency cases as illustrated by an upper ablephary causing corneal exposure or optic nerve compression by cranio-stenosis that may be an indication of an extremely urgent cranio-orbito facial surgery, we do have the choice between an early surgery and a delayed one. Given the facts that surgical techniques are becoming more and more sophisticated, and that anaesthesia and resuscitation are making much progress, the trend is to operate very early to avoid functional sequellae and psychological disorders for the child and his family.

The indication for such early surgery will of course depend on the importance of the lesion, its evolution, the demand of the family and the calculated risks as compared to those for a delayed type of surgery.

Technically, early surgery of soft tissues or bony parts does not seem more difficult than surgery in adolescence. In some cases, delayed surgery (planned toward the end of the growing period), will appear more appropriate. We also believe that it is preferable to perform orbital enlargement osteotomies on well-grown orbits rather than on young ones.

\section{What surgical tactic to use?}

This decision will depend on the clinical and investigatory findings.

In isolated forms, the treatment is simple and is directed towards a single symptom, whereas in multi symptomatic forms, one must plan several surgical procedures done during one or more surgical intervention. They may include orbital osteotomies, palpebral lengthening or correction of a pal- 
pebral coloboma as well as reformation of a conjunctival fornix by a mucosal graft.

As a general rule, bone will be treated first, followed by soft tissue surgery. The surgical treatment must be carefully and precisely planned as several operative settings may be often needed.

In many cases, surgery will be 'border-line', calling for the assistance of several teams: ophthalmic, plastic, cranio-maxillo-facial and neurosurgical specialists will have to cooperate.

\section{Indications and Methods According to Anatomo-clinical Forms}

A-Eyelids Malpositions:

\section{1-Entropion}

Entropion is characterised by turning in of the eyelid margin causing irritation of the eye and cornea. It must be distinguished from epiblepharon that is an abnormal fold of skin occurring in young children that retracts over the lower lid, pressing the lashes inwards.

The principle of treatment is to excise an ellipse of skin and underlying hypertrophied orbicularis muscle lelow the inferior punctum. The skin edges are sutured to the lower border of the tarsus to prevent the orbicularis from again overriding the lid margin. ${ }^{3}$

\section{2-Ectropion}

The congenital ectropion syndrome ${ }^{4}$ is a rare congenital deformity, with shortage of skin in all 4 lids sometimes along with a degree of ptosis but in which although there is quite a marked telecanthus there are no epicanthal folds of any sort.

The treatment includes extensive post-

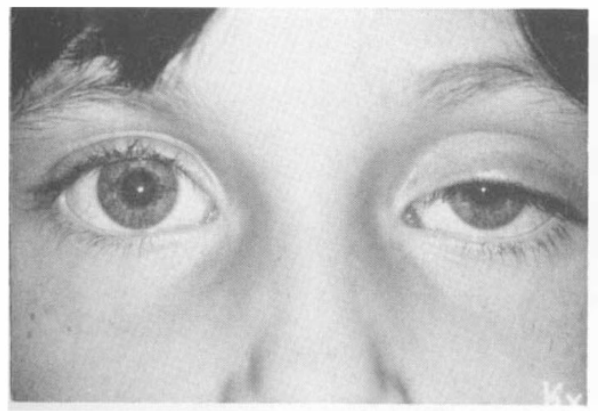

Fig. 3. a-Moderate left congenital ptosis. Moderate levator function. auricular full thickness skin grafts inserted into the lower lid via a para-marginal incision. The orbicularis muscle must be split just above the lower lateral orbital margin and the orbital septum divided with scissors along the inferior orbital margin. The upper lids will be reconstructed after an interval of 6 months by insertion of adequate split-skin grafts or thin full-thickness grafts, by a para marginal incision.

\section{3-Ptosis (Fig. 3)}

Congenital ptosis 5 is due to a dystrophy of the levator muscle. In some cases, there is an aponeurotic defect and nerve palsy. The management of ptosis depends on the diagnosis and examination findings. Levator resection by an anterior approach is the method of choice. The importance of the resection depends on the degree of levator function. In moderate ptosis with good levator function (more than $10 \mathrm{~mm}$ ), the Fassanella-Servat procedure is a good one. The upper border of the tarsus is excised with the lower part of Muller's muscle and overlying conjunctiva. When the levator function is poor or when the levator muscle cannot be used, a brow suspension by connecting the frontalis muscle and eyebrow to the eyelid with a subcutaneous sling must be performed (Fig. 4).

\section{4 -Levator eyelid retraction ${ }^{6.7}$}

This congenital deformity is a rare condition with a true levator eyelid retraction sometimes associated to shortage of skin.

This upper lid retraction can be treated by

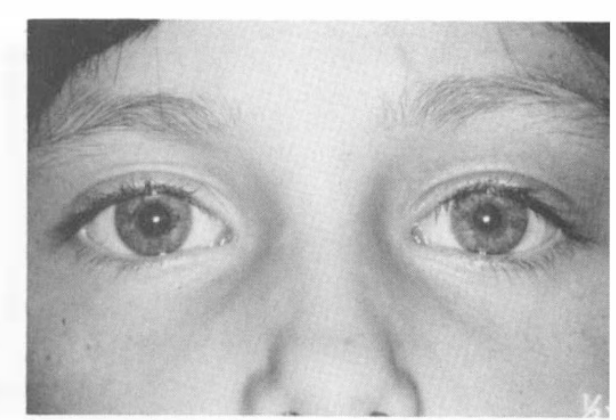

$b$-Result after large levator shortening. 


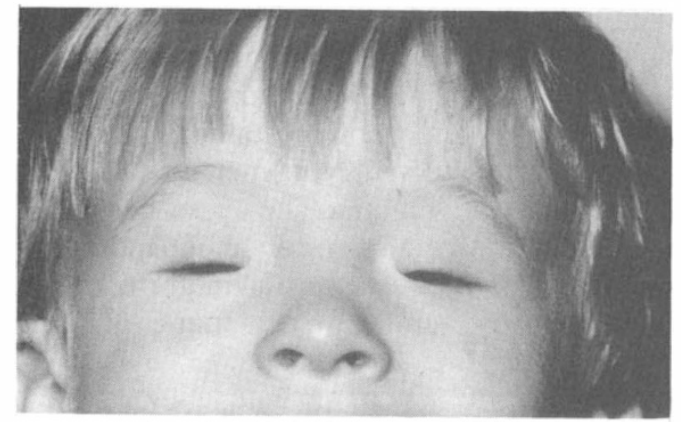

Fig. 4. a-Severe blepharophimosis with ptosis, epicanthus, telecanthus.

lengthening of the levator muscle by the following technique (Fig. 5).

Incision through the skin of the upper lid in the palpebral fold, opening of the septum, complete section of the levator muscle at the upper border of the tarsal plate, lengthening of the muscle by interposition of lyophilised sclera or a strap of synthetic material (GoreTex) at the upper border of the tarsus. This lengthening must equal twice the excess retraction of the upper lid. Slight upper lid retraction can be treated by Müller's muscle resection.

\section{5-Medial canthus malpositions ${ }^{8.9}$}

Congenital telecanthus may be encountered either as a primary condition in which the interpupillary distance itself remains within normal limits, or it may be a secondary condition associated with an increase of the interpupillary distance, indicating in case of absence of strabismus an increased distance between the eyeballs and the underlying bony orbits.

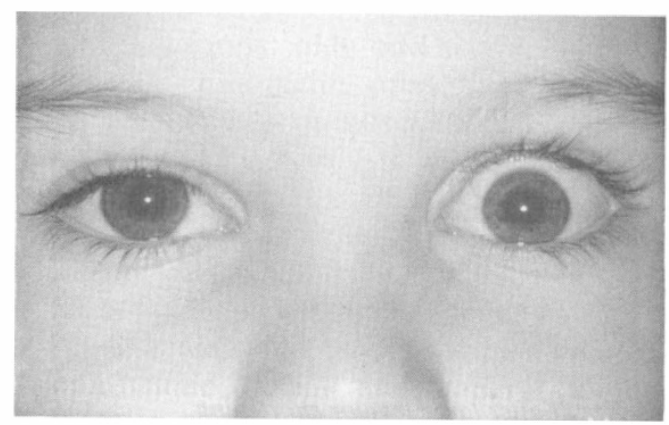

Fig. 5. a-Left congenital levator eyelid retraction.

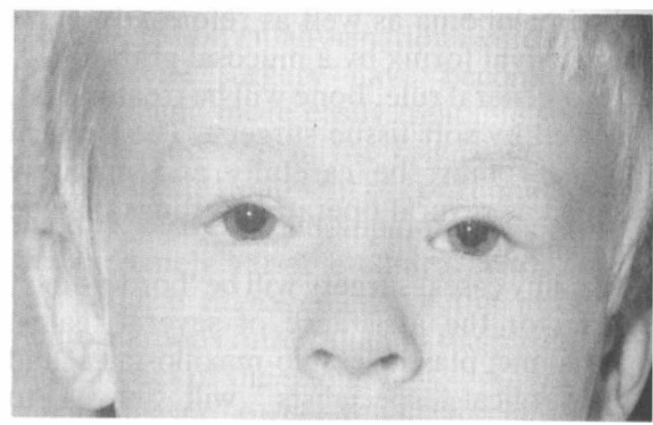

b-After correction of the epicanthus-telecanthus and bilateral suspension with autogenous fascialata.

In the latter condition, the telecanthus or the telorbitism is corrected when the orbital contents and the orbit themselves are moved into a normal position.

The important points in correction of congenital telecanthus are to arrange for the accommodation of the excess soft tissue in the area by means of transposed flaps, to shorten the medial canthal tendons, or in severe cases, to attach the medial canthal tendons permanently to each other across the nasal cavity by means of stainless steel wire.

The design of the flaps is established by first marking a point where the medial canthus ought to lie and then, after drawing the nasal skin medially so as to obliterate any fold present, marking the site of the actual canthus.

These 2 salient points are joined and from the centre a line is drawn, slightly shorter than and at $60^{\circ}$ to the original line.

Backcuts of the same length are drawn at $45^{\circ}$, and the design of the four quadrilateral flaps is completed by marking in paramarginal lines again of the same length as the verticals.

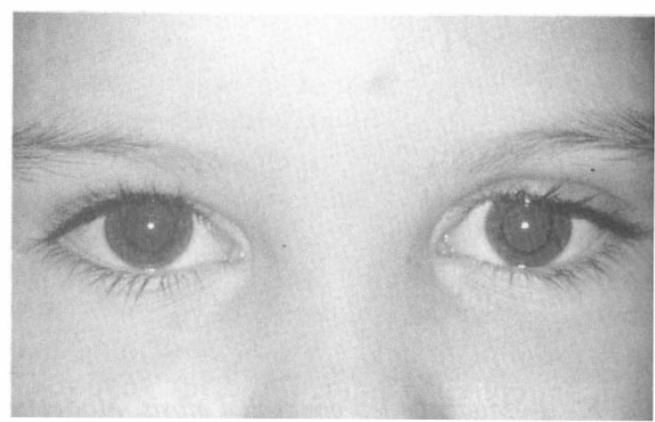

b-Result after levator recession and scleral graft. 
The flaps are incised and raised along with some underlying orbicularis muscle, and the elongated superficial component of the medial canthal tendon is rejected, and a 4-0 nonabsorbable suture is inserted through the stump of the tendon close to the canthus and through the periosteum underlying the medial stump. When this suture is tied, it draws the medial canthus nasally, and the flaps are then easily transposed. Since they are quadrilateral, the flaps may need to be trimmed to fit the triangular defects, and they are then sutured into place using 6-0 chromic catgut (which avoids the need to remove the sutures 4 or 5 days later). The scars of the transposed flaps are surprisingly obvious for several months but eventually become practically invisible.

When telecanthus is present alone without any accompanying folds, as in Waardenburg's syndrome, exactly the same formula is used, but no obliteration of folds has to be carried out before designing the flaps. Because of this, the whole of the flap design lies exposed, but the technique is carried out exactly as before (Fig. 6).

A much more severe type of telecanthus is encountered in blepharophimosis where there may be up to $20 \mathrm{~mm}$ of increase of the intercanthal width. In blepharophimosis, along with the telecanthus there are small inverted epicanthal folds, a severe ptosis which is most difficult to correct by levator surgery alone, and an absence of laxity of the skin of the lids in a vertical direction, which may amount to actual ectropion in the outer half of the lower lid. In these severe cases with telecanthus and epicanthal folds, the treatment includes a skin

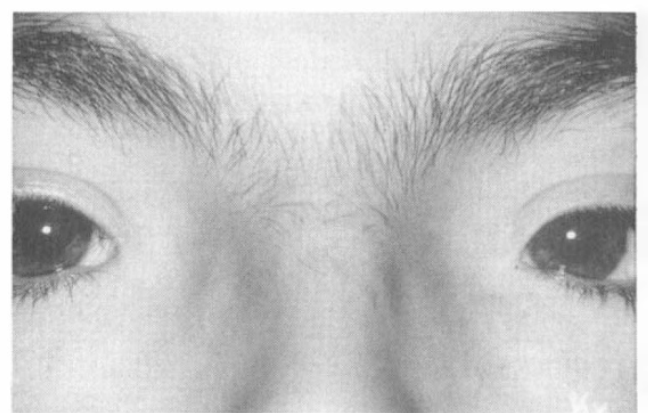

Fig. 6. a-Severe Waardenburg's syndrome with telecanthus. plasty by four quadrilateral flaps or a V-Y plasty, a reduction of the thickness of the anterior lacrimal crest and medial orbital bony wall, and a medial canthopexy by a transnasal wire fixation. ${ }^{8.9}$

\section{6-Congenital eyelid colobomas ${ }^{10}$}

Congenital colobomas are clefts of varying configuration found in the visual system. They can be found in all areas of the lids. More than one lid may be involved in the same patient, or there may be multiple colobomas in the same lid. They most often occur as isolated anomalies, but are sometimes seen in patients with other congenital lesions, and ocular lesions, the most common being dermoïds and dermolipomas, facial lesions as other facial clefts.

The aetiology of eyelid colobomas is not clear. The predominant aetiologic theory incriminates the mechanical action of amniotic bands. Heredity does not seem to play a significant role in most colobomas except in the lower-lid colobomas associated with mandibulo-facial dysostosis (FrancheschettiTreacher-Collins syndrome).

Recently, the colobomas of the eyelids have been classified with the new anatomic classification of facial clefts. ${ }^{2}$

Treatment of congenital colobomas will depend on the extent of the defect as well as its position. ${ }^{10.11}$ It is possible primarily to close defects of up to one-third of the lid. The rounded edges of the coloboma are squared by a razor knife, creating fresh new surfaces to help ensure the most precise closure. After the edges of tarsus are brought together by placing 6-0 chromic sutures in the pretarsal fascia, attention is focussed on the lid margin

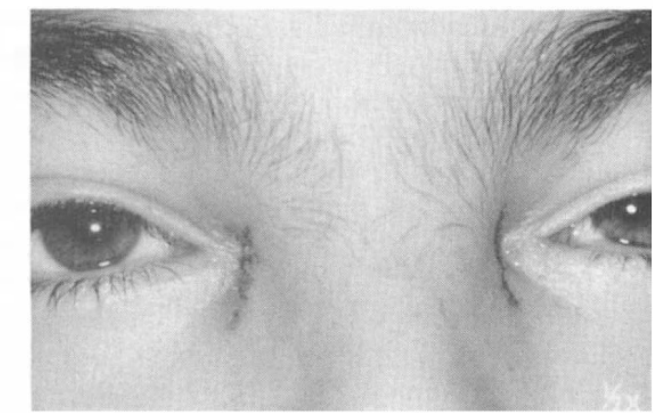

$b$-Result after medial canthopexy by a transnasal wire fixation. 
which is aligned with three interrupted 6-0 silk sutures. The remainder of the skin is then closed with interrupted 6-0 silk sutures.

In some patients with slightly larger defects, the horizontal tension may still be too great so that a cantholysis must be performed. By cutting either the upper or lower wing of the lateral canthal tendon, depending on which lid is being repaired, the surgeon will have several more millimeters of tissue with which to work.

Colobomas involving much more than onethird of the lid will require a more extensive lid reconstruction procedure. Upper-lid defects must be repaired by using a composite graft from either the other upper lid or the opposing lower lid, ${ }^{12}$ a Mustardé rotation flap,,$^{13}$ or a Cutler-Beard bridge flap from the opposing lower lid..$^{14}$ Extensive defects of the lower lid are best required by a large cheek rotation flap ${ }^{13}$ or an orbito-naso-genial flap ${ }^{15}$ with a chondro-mucosal graft for the posterior lamella, or a tarso-conjunctival flap from the opposing upper lid doubled by a skin graft for the anterior lamella. ${ }^{11}$

Frequently congenital eyelid colobomas are associated with adnexal dermoïds. ${ }^{16}$ Dermoïd cysts are common congenital anomalies found in the brow, the eyelid or the orbit. Solid dermoïd tumors on the limbus and dermolipomas in the fornices are much less common than dermoïd 'cysts. Complete operative removal is the treatment of choice in all dermoïd tumors; however there are times when the needs of the patient are best served by a subtotal excision.

A partial resection is recommended rather than endangering vital orbital structures.

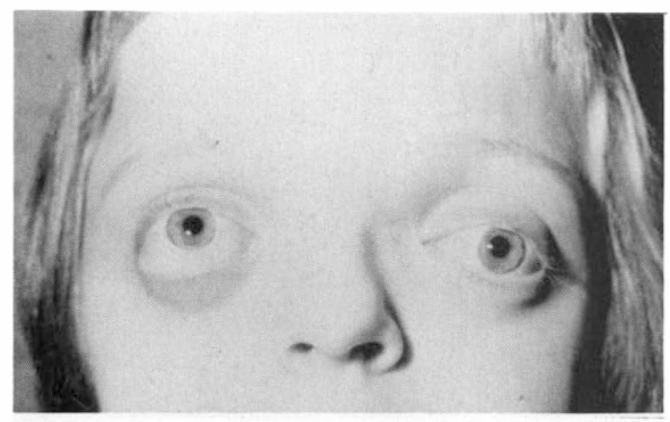

Fig. 7. a-Craniofacial stenoses, Crouzon's disease with exophthalmos and strabismus.
In some cases after excision of the lesion, a reconstruction of the defect must be performed by conjunctival flaps or mucosal grafts.

\section{B-Congenital Orbital Abnormalities ${ }^{16.17}$}

Congenital orbital abnormalities can be classified as congenital anophthalmos and microphthalmos as orbital dystopias or plagiocephalies due to a cranio facial cleft, or as hemicranial stenoses, cranio facial stenosis as the Crouzon and Apert syndromes, or hypertelorism and finally as craniofacial and lateral clefts. Cranio facial stenosis affect the sagittal diameter in Crouzon and Apert syndromes, the vertical diameter is abnormal in orbital dystopia, and the transverse diameter is affected in hypertelorism. The general principle of treatment is to displace the functional part of the orbit, that is, that part of the orbit that carries the globe. This part consists of the orbital walls situated behind the equator of the eyes.

\section{1-Craniofacial stenosis ${ }^{18}$ (Fig. 7)}

The Crouzon and Apert syndromes are characterised by stenosis of all the sutures of the upper part of the skull and consequently intracranial hypertension, papillary oedema and sometimes blindness.

The facial characteristics are the reduced size of the facial structures, exophthalmos and exotropia. These may be combined with nasopharyngeal atresia and deformity of the maxilla. The aims of the operation are to give the central portion of the face a normal profile, to re-establish dental occlusion, and to increase the depth of the orbit.

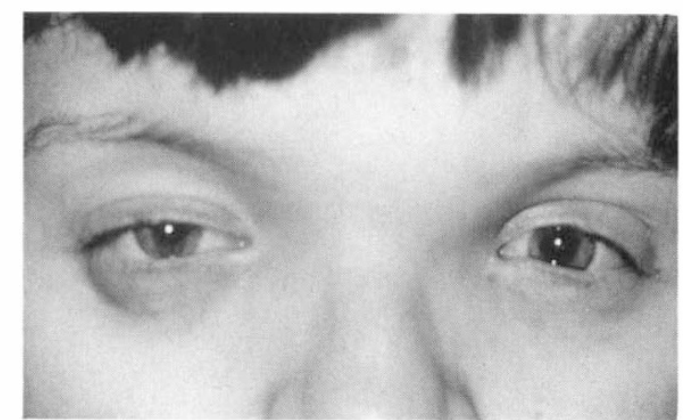

b-Result after Tessier-Lefort III osteotomy (surgeon Dr P Tessier) and strabismus surgery. 
The method used is a cranio-facial dysjunction which is both interfronto-facial and interpterygomaxillary, and to re-establish the vertical dimensions of the face. If possible, respiratory pathways have to be restored.

\section{Procedure:}

The total osteotomy of the central portion of the face is a complex operation consisting of several stages. We describe the mid-facial advancement by the Tessier-Lefort III type osteotomy.

A coronal incision has many advantages in facilitating the osteotomies along the frontomalar region. One follows the lateral surface of the orbit into the retromalar space and performs a temporo-zygomatic dysjunction. Then on the frontal bone, one can make either a deep vertical frontal spur or a transverse frontal crescent, behind which bone grafts can be placed to prevent relapse.

Along the fronto-naso-orbital region, one first carries out a vertical section of the medial walls down to the floor, $10 \mathrm{~mm}$ behind the posterior lacrimal crests. Then, centrally, an infra-basilar osteotomy is made parallel to the stage of the anterior cranial base. Subsequently, an inter-pterygo-maxillary dysjunction is performed by an oral approach with a curved $8 \mathrm{~mm}$ osteotoma.

Dysjunction is performed with the Rowe forceps or with right-angles grapnels, introduced behind the maxilla. The disengagement should be carried out to an overcorrection of at least $10 \mathrm{~mm}$. Consequently, seven gaps occur, a central fronto-nasal gap, two lateral gaps and two retromalar gaps, which should all be closed with bone grafts.

These grafts must support the repositioning of the face and, in particular must prevent any backward relapse into the original position. In addition, the grafts have to close the fissures in the orbital walls and are used to correct the profile of the glabella, of the supra-orbital ridge, of the zygomatic arch and of the infraorbital rim.

The osteosynthesis finally has to provide the permanent fixation of the reconstructed region. As regards the dental occlusion, there is a clearcut difference in procedure between adults and children.

In the adult one establishes only normal occlusion, but in the child, sagittal over-correction of $6-8 \mathrm{~mm}$ is necessary in order to provide space for future dental eruptions and orthodontics. The intermaxillary fixation is used only as a means of rectifying the final articulation. The central position of the face remains without support in its new position without the aid of intermaxillary fixation.

\section{2-Congenital orbital dystopias or orbital plagio cephalies:}

When there is orbital dystopia, which is always the consequence of other malformations, one must look for the causal malformation and its repercussions.

The operation consists of the monobloc raising of the orbit by a frontal and malar osteotomy, followed by repositioning in the proper position. In addition, hypoplastic and displaced parts should be remodelled by bone grafts to smooth the exterior aspect. The monobloc raising of the orbit should preferably be done by elevating it in its totality, in one or two fragments. Again the coronal incision is used. The cranial base is exposed from the crista galli back to the posterior border of the lesser wing. Oblique or step osteotomies are traced on the frontal and on the malar bone to ensure that the orbit is mobilised as a monobloc.

The inferior facial osteotomy is done at two different levels. Laterally, it is oblique and located relatively high on the malar bone. Medially it is transverse and directed towards the inferior meatus.

During the repositioning one has to keep in mind that transverse displacement corrects hypertelorism and telecanthus. Anterior displacement may result in exophthalmos if the defects are not sufficiently occluded by bone grafts. By relation of the bloc, the lateral quadrants can be elevated and the malar bone may be advanced. The position of the orbital rim can be adjusted. If a unilateral repositioning is done, the contralateral orbit should be used as a reference point.

\section{3-Hypertelorism (Figs. 8-9):}

Hypertelorism is a congenital malformation characterised by an abnormally exaggerated distance between the two orbits and, there- 


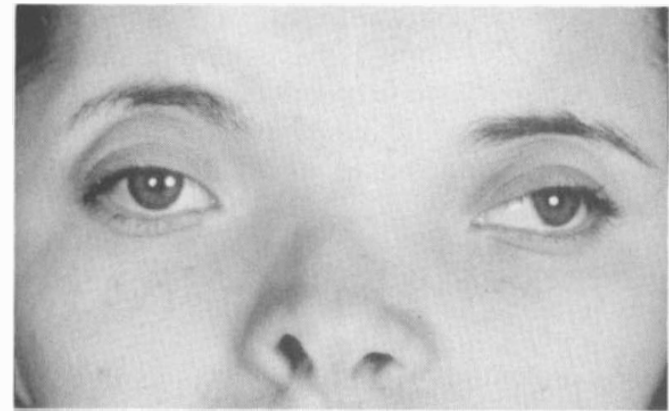

Fig. 8. a-Hypertelorism with a craniostenosis in the interorbital space.

fore, between the two ocular globes, with a cranio facial cleft or a craniostenosis in the interorbital space. When hypertelorism is secondary to a craniostenosis, there is always a brachycephaly and an elevation of the level of pterion and generally a bifid nose.

The abnormal appearance is much less the consequence of hypertelorism than of the malformations which cause the hypertelorism. To correct these malformations of the interorbital space, one has to restore the interorbital distance to their normal dimensions. The usual procedure by the intracranial route consists of the displacement of the orbit transversely by a total osteotomy of the frame work of the walls. A central resection is carried out on the frontal bone on the bones of the nose,

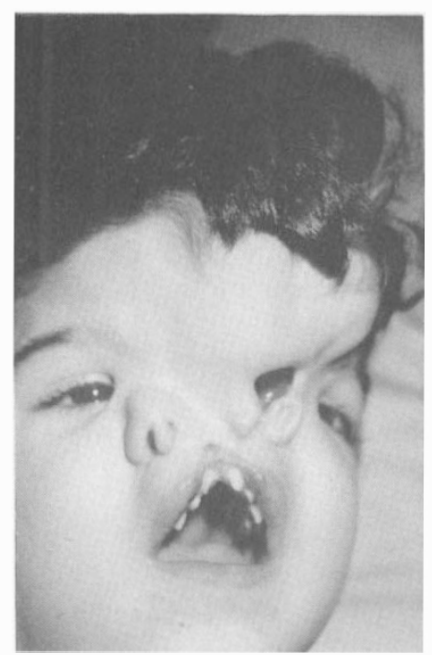

Fig. 9. a-Hyertelorism with craniofacial cleft.

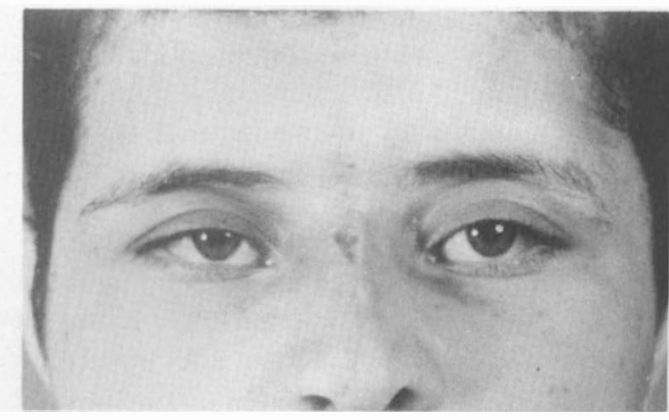

b-Result after displacement of the orbit transversely (Surgeon Dr P. Tessier).

on the fronto maxillary processes and on the ethmoïd bone. The anterior resection is completed by resection of the lateral masses of the ethmoïd and of the structures within the nose. The septum, vomer, inferior and medial turbinates and the edges of the piriform aperture are severed. With medial canthopexies, the orbits are brought to the midline and the aponeurosis of the temporal muscle is advanced to the lateral pillar of the orbit and to the temporal crest. Finally the nasal bridge and possible defects have to be grafted with bone. If hypertelorism and orbital dystopia have to be treated, the cranial approach makes it possible to correct the transverse as well as the vertical displacement of the orbit. If it concerns orbital dystopia in the Crouzon or the Apert syndromes the craniofacial stenosis are often asymmetric, although in a certain way they are plagiocephalies. Although these facial retrusions are generally approached by an extracranial route, considerable orbital dystopia can be an indication

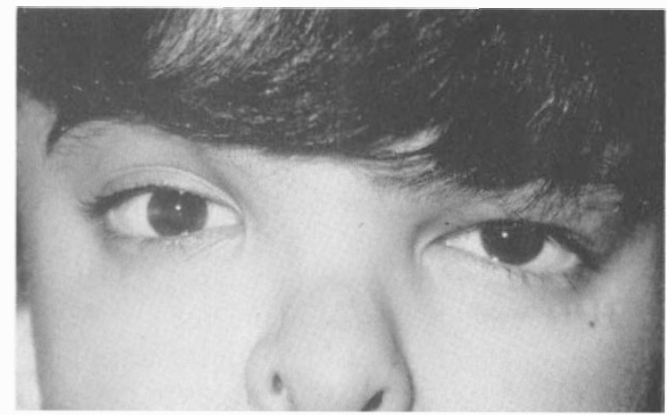

b-Result after two major operations (Surgeon Dr P. Tessier). 
for simultaneous elevation of the orbit by the intracranial route. If hypertelorism and craniofacial stenosis go together, the cranial route should be combined with ethmoidal resection.

Craniotomy must be done by the neurosurgeon as soon as intracranial hypertension is diagnosed. But in cases of facial deformity the primary craniotomy ought to be arranged according to what will be done in the future, either on the orbitofrontal region or on the facial abnormalities. The ages for the first surgical stage should preferably be between three and five years.

Our experience in the surgery of craniofacial stenosis has demonstrated that the first operation always has the best chance. Secondary revisions in the orbitocranial area are much more difficult and hazardous than the primary operation. Consequently, the planning for the primary intervention should aim at a complete redesigning of the facial architecture.

In operations for hypertelorism, secondary operations are unavoidable. These involve restoration of palpebral occlusion and the closure of maxillo palatine clefts, and rarely involve reinforcement of the dura. The three operations are satisfactory in hypertelorism secondary to cranial or facial clefts. In craniofacial stenosis and orbital dystopias, aesthetic as well as functional objectives should be pursued to correct the nose, chin, lateral canthus, dental occlusion, and motility disorders such as ptosis and strabismus.

We advocate that no corrective strabismus surgery is undertaken until at least six months after the major orbital translocation procedures, by which time the strabismus is

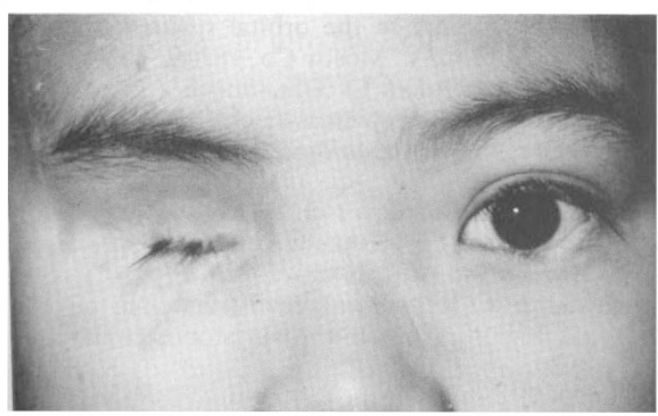

Fig. 10. a-Severe congenital microphthalmos. stabilised. We have, in fact, observed that medial orbital translocation in teleorbitism and sagittal orbital translocation in craniofacial stenosis induce a reduction of the orbital divergence and so a reduction of the exotropia. Orbital surgery can also induce an oculomotor disturbance that did not exist before. ${ }^{20,21}$

This recommendation to wait for strabismus surgery can be modified depending on the age of the patient when examined and when the orbital translocation was performed.

If the malformation is discovered very early and surgery takes place in the first years (between 3 and 5 years for some authors), the strabismus will be treated and operated on later. If the malformation is to be treated later, after the eruption of permanent dentition or for any other reason, the strabismus surgery should be performed first for functional reasons.

We think that early correction of congenital deviation is more likely to result in attainment of binocularity. However, we know that after craniofacial reconstruction decompensation of strabismus is possible, especially when binocular vision is absent and revision of strabismus surgery is frequent.

If the malformation is seen and operated on in adults strabismus surgery will be done after orbital surgery because treatment is undertaken for only cosmetic reasons.

\section{5-Anophthalmos and severe congenital microphthalmos ${ }^{22}$ (Fig. 10)}

In patients with congenital anophthalmos and severe microphthalmos, we are faced with a tiny orbit and socket and with little eyelids,

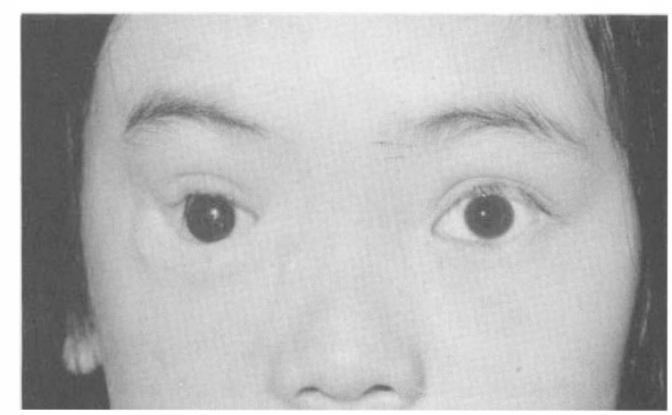

b-Result after orbital osteotomy, socket expansion, inferior eyelid reconstruction, prosthesis. 
frequently preventing retention of a standard conformer or prosthesis. Socket expansion is sometimes impossible with micro-orbitism, the retention of a prosthesis is also difficult when malformation of the eyelids exists. The treatment in these difficult cases includes, in the first stage, an orbital expansion which depends on the cephalometric studies of the patient: transverse osteotomy of the maxilla and zygomatic bone with lateral bar via an extra-cranial approach, vertical osteotomy of the roof of the orbit via an intracranial approach. In some cases, the osteotomy will include expansion in the transverse and vertical diameter with bone grafts in the defects and on the lateral and superior rims. At the same time, socket expansion will be performed by incision of the conjunctival sac circumferentially, with mucosal or split skin grafts on a conformer. The second stage includes eyelid reconstruction by different flaps. A third stage is frequently needed for correction of eyelid malpositions on the prosthesis: ptosis, entropion surgery.

\section{6-Cryptophthalmos ${ }^{23}$ (Fig. 11)}

The cryptophthalmos syndrome is a very rare congenital defect with incomplete or complete failure in the development of one or both eyelids, skin covering the anterior segment. The eyeball is anatomically present but not functional. The orbital cavity is well developed. The surgical treatment includes a socket reconstruction with eyeball conservation if possible. At the same time, or later, the eyelids are reconstructed by different flaps.

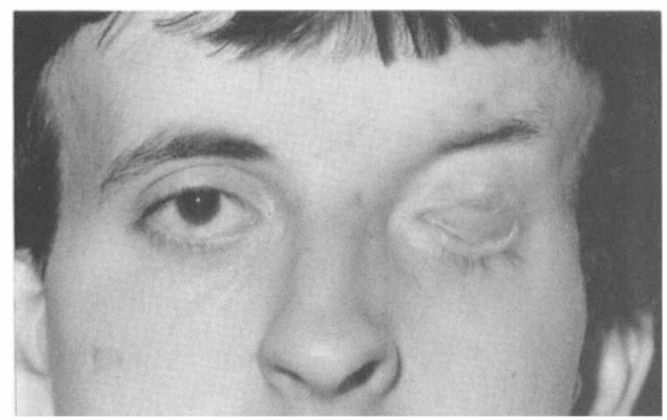

Fig. 11. a-Partial crvptophthalmos.

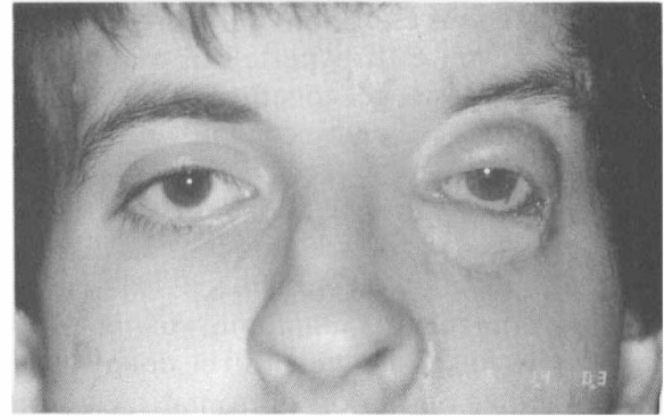

b-Result after socket reconstruction, eyelid reconstruction, prosthesis on the eyeball.

\section{References}

${ }^{1}$ Tessier P, Guiot G, Rougerie J, Delbet P, Pasteriza P: Osteotomies cranio-naso-orbito-faciales. Hypertélorisme. Ann Chir plast 1967, 12: 86.

2 Tessier P: Anatomical classification of facial, craniofacial, and laterofacial clefts in Symposium on plastic surgery in the orbital region. St Louis 1976, The C. V. Mosby Co, 189-98.

${ }^{3}$ Collin JR: Congenital entropion in 'Manual of systematic eyelid surgery'. Churchill Livingstone, 1983, 16.

${ }^{4}$ Mustarde JC: Congenital ectropion syndrome. In 'Repair and Reconstruction in the orbital region'. Churchill Livingstone, 1980, 351-5.

${ }^{5}$ Morax S and Ruban JM: Les ptosis. J Fr ophthalmol. (In Press).

${ }^{6}$ Morax S and Hurbli T: Choice of surgical treatment for Graves' disease. J cranio-max Fac Surg 1987, 15: $174-80$.

${ }^{7}$ Morax S: Traitement des rétractions palpébrales par allongement du muscule releveur. Ann Chir Plast Esthet 1983, 28: 361-8.

${ }^{8}$ Mustarde JC: Epicanthal folds and the problem of telecanthus. Trans Ophthalmol Soc UK 1963, 83: 397-411.

${ }^{9}$ Mustarde JC: Telecanthus in 'Third international symposium of plastic and reconstructive surgery of the eye and adnexia'. Williams and Wilkins, Baltimore 1982, 199-205.

${ }_{10}$ Wilkins RB and Carroll RP: Adnexal dermoids and congenital eyelid colobomas in 'Symposium on plastic surgery in the orbital region'. St Louis 1976, The C. V. Mosby Co, 187-8.

${ }^{11}$ Morax S and Benia L: Traumatismes et chirurgie plastique des paupières. Encycl Med Chir (Paris France). Ophthalmologie, 21100 D 10, 11-1985, 18.

12 Hubner H: Kolobomverschluss Mittels fre er Tarsus lidrandüberf Lanzung. Klin Monatsbl, Augenheilk 1976, 168: 677-82.

${ }^{13}$ Mustarde JC: Repair and reconstruction in the orbital region. Churchill Livingstone, Edinburgh, 1980.

${ }^{14}$ Cutler ML and Beard C: A method for partial and total upperlid reconstruction. Am J ophthalmol 1955, 39: 1 . 
${ }^{15}$ Tessier R: Blepharopoièses inférieures. Bull Mem Soc Fr Ophtalmol 1960, 73, 213-54.

${ }^{16}$ Rougier J, Tessier R, Hervouet F, Woillez M, Lekieffre M, Derome P: Colobome's palpébraux, dermoïdes épibulbaires et du fornix. Ectropion congenital in 'Chirurgie plastique orbito palpébrale. Rapport Soc Fr Oph Paris 1977, Masson éd, 209-22.

${ }^{17}$ Morax S: Correction of congenital orbital abnormalities. Orbit 1983, 2: 121-8.

${ }^{18}$ Tessier P: The definitive plastic surgical treatment of the severe facial deformities of cranio-facial dysostosis. Crouzon's and Apert's syndromes. Plast reconstr Surg 1971, 48: 419.

${ }^{19}$ Tessier P: Orbital hypertelorism. Scand J plast reconstr Surg 1972, 6: 135.
${ }^{20}$ Morax S: Oculomotor disorders in cranio-facial malformations. In 'Cranio-facial surgery' Boston 1985. Little, Brown and Company edited by Caronni P., 97-107.

${ }^{21}$ Morax S: Changing of the eye position after craniofacial surgery in 'Cranio-facial surgery' Boston 1985. Little, Brown and Company edited by Caronni P., 200-10.

${ }^{22}$ Morax S: Reconstruction orbito-palpébrale au cours des anophtalmies et microphtalmies sévères congénitales. Ann Chir Plast Esthét 1987, 32: 255-61.

${ }^{23}$ Morax S and Herdan ML: Reconstruction orbito palpébrale au cours de deux cas de cryptophtalmies partielles. Ann Chir Plast Esthet 1987, 32: 319-26. 